\section{AB011. Linkage strategies for successful and sustainable partnerships: a practical framework for community engagement by palliative care services}

\author{
John Rosenberg \\ NHMRC CRE for End of Life Care, Institute of Health and \\ Biomedical Innovation, Queensland University of Technology, \\ Brisbane, QLD, Australia
}

Abstract: Partnerships are central to the success of linkages between palliative care services and the communities they support. The goal of partnership is to achieve more than individuals and groups can achieve on their own, yet the concept is often poorly understood. A clearly articulated understanding of partnership is a powerful step in transforming an organization's engagement with the community. The aim of this workshop is to enable participants to gain a clear understanding of partnership, understand the recognized evidence-based principles of establishing and maintaining partnerships, and identify practical approaches to partnering to take back to their organizations and communities.

Keywords: Palliative care; organizations; community

doi: 10.21037/apm.2018.s011

Cite this abstract as: Rosenberg J. Linkage strategies for successful and sustainable partnerships: a practical framework for community engagement by palliative care services. Ann Palliat Med 2018;7(Suppl 1):AB011. doi: 10.21037/apm.2018.s011 